\title{
Charm-baryon enhancement and charm fragmentation fractions in small systems measured with ALICE
}

\author{
Jianhui $\mathrm{Zhu}^{1,2, *}$ for the ALICE Collaboration \\ ${ }^{1}$ Institute of Particle Physics, Central China Normal University, 152 Luoyu Road, 430079 Wuhan, China \\ ${ }^{2}$ GSI Helmholtz Centre for Heavy Ion Research, Planckstraße 1, 64291 Darmstadt, Germany
}

\begin{abstract}
Recent measurements of charm-baryon production at midrapidity by the ALICE collaboration show baryon-to-meson yield ratios significantly higher than those measured in $\mathrm{e}^{+} \mathrm{e}^{-}$collisions, suggesting that the charm fragmentations are not universal across different collisions systems. Thus, measurements of charm-baryon production are crucial to study the charm quark hadronisation in proton-proton $(\mathrm{pp})$ collisions. In proton-lead $(\mathrm{p}-\mathrm{Pb})$ collisions, the measurements of charm baryons provide important information about cold nuclear matter effects and help to understand how the possible presence of collective effects could modify the production of heavy-flavour hadrons. In this contribution, the most recent results on open charm-hadron production in $\mathrm{pp}$ and $\mathrm{p}-\mathrm{Pb}$ collisions measured by ALICE are discussed.
\end{abstract}

\section{Introduction}

The production of heavy-flavour hadrons in high-energy hadronic collisions can provide important tests of the theory of quantum chromodynamics (QCD). The production cross sections of heavy-flavour hadrons can be calculated using the factorisation approach as a convolution of three factors [1]: the parton distribution functions (PDFs) of the incoming nuclei, the hard-scattering cross section at partonic level, calculated as a perturbative series in powers of the strong coupling constant $\alpha_{\mathrm{s}}$, and the fragmentation functions of heavy quarks into corresponding heavy-flavour hadrons, which is an inherently non-perturbative process related to, or even driven by, the confining property of QCD. The heavy-flavour baryon-to-meson ratio is an ideal observable related to the hadronization mechanism since the contributions from parton distribution function and parton-parton scattering terms cancel in the ratio. The $\Lambda_{\mathrm{c}}^{+} / \mathrm{D}^{0}$ ratio at the LHC is enhanced with respect to predictions based on $\mathrm{e}^{+} \mathrm{e}^{-}$and ep experiments, suggesting that the charm fragmentation functions are not universal among different collision systems. Several hadronization mechanisms, such as colour reconnection (CR) beyond the leading colour approximation [2], coalescence [3,4] and feed-down from a largely augmented set of higher mass charm-baryon states beyond the current listing of the particle data group (PDG) [5], have been proposed to explain this enhancement. The newest measurements of the charm baryons $\Lambda_{\mathrm{c}}^{+}, \Sigma_{\mathrm{c}}^{0,++}, \Xi_{\mathrm{c}}^{0,+}$ and $\Omega_{\mathrm{c}}^{0}$ performed with the ALICE experiment will be used to verify predictions from these hadronization mechanisms.

\footnotetext{
*e-mail: jianhui.zhu@cern.ch
} 

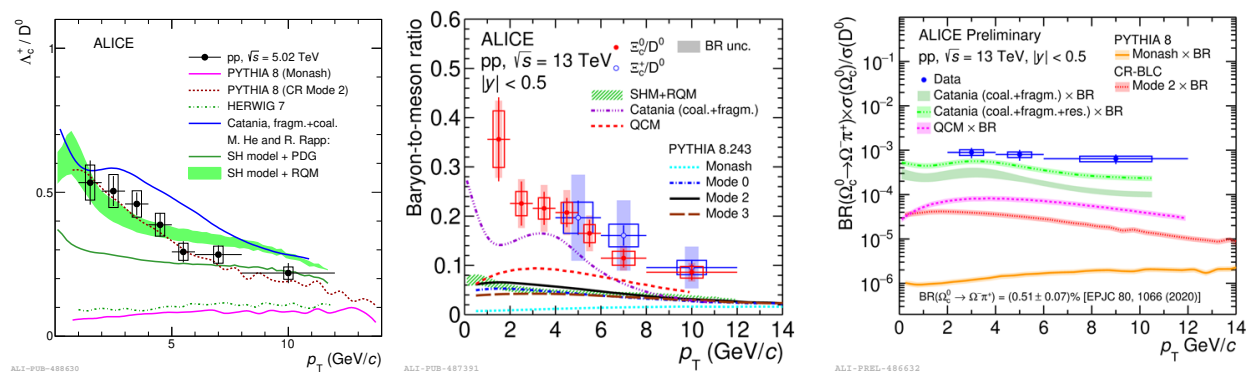

Figure 1. Left: The $\Lambda_{\mathrm{c}}^{+} / \mathrm{D}^{0}$ ratio as a function of $p_{\mathrm{T}}$ measured in pp collisions at $\sqrt{s}=5.02 \mathrm{TeV}$ [7]. Middle: The $\Xi_{\mathrm{c}}^{0} / \mathrm{D}^{0}$ and $\Xi_{\mathrm{c}}^{+} / \mathrm{D}^{0}$ ratios measured in pp collisions at $\sqrt{s}=13 \mathrm{TeV}$ [8]. Right: The $\mathrm{BR} \times \Omega_{\mathrm{c}}^{0} / \mathrm{D}^{0}$ measured in pp collisions at $\sqrt{s}=13 \mathrm{TeV}$. All the charm baryon-to-meson yield ratios are compared to theoretical calculations $[2-5,11]$.

\section{Charm baryon-to-meson yield ratios in pp collisions}

Thanks to the large data sample collected during the Run 2 period in pp collisions at $\sqrt{s}=5.02$ and $13 \mathrm{TeV}$, ALICE measured all the ground-state charm hadrons down to low transverse momentum $\left(p_{\mathrm{T}}\right)$, including charm mesons $\left(\mathrm{D}^{0}, \mathrm{D}^{+}, \mathrm{D}_{\mathrm{s}}^{+}[6]\right)$ and charm baryons $\left(\Lambda_{\mathrm{c}}^{+}[7], \Xi_{\mathrm{c}}^{0,+}[8,9]\right.$, and $\left.\Omega_{\mathrm{c}}^{0}\right)$. The $\Lambda_{\mathrm{c}}^{+} / \mathrm{D}^{0}$ yield ratio is measured as a function of $p_{\mathrm{T}}$ in pp collisions at $\sqrt{s}=5.02 \mathrm{TeV}$ as shown in Fig. 1 (left), the $\Xi_{\mathrm{c}}^{0} / \mathrm{D}^{0}$ and $\Xi_{\mathrm{c}}^{+} / \mathrm{D}^{0}$ yield ratios are measured in pp collisions at $\sqrt{s}=13 \mathrm{TeV}$ as shown in Fig. 1 (middle). For $\Omega_{\mathrm{c}}^{0}$, the absolute decay branching ratio (BR) of $\Omega_{\mathrm{c}}^{0} \rightarrow \Omega^{-} \pi^{+}$is not measured, hence the BR of $\Omega_{\mathrm{c}}^{0} \rightarrow \Omega^{-} \pi^{+}$times cross section of $\Omega_{\mathrm{c}}^{0}$ over cross section of $\mathrm{D}^{0}$ in pp collisions at $\sqrt{s}=13$ $\mathrm{TeV}$ is reported in Fig. 1 (right). In order to compare data with models, a theoretical calculation of $\operatorname{BR}\left(\Omega_{\mathrm{c}}^{0} \rightarrow \Omega^{-} \pi^{+}\right)[10]$ is used to multiply different models. The $\Lambda_{\mathrm{c}}^{+} / \mathrm{D}^{0}$ and $\Xi_{\mathrm{c}}^{0} / \mathrm{D}^{0}$ ratios show a downward trend with increasing $p_{\mathrm{T}}$. The Monte Carlo generator PYTHIA8 (Monash) [11] tuned on measurements in $\mathrm{e}^{+} \mathrm{e}^{-}$collisions largely underestimates all four charm baryon-to-meson yield ratios, providing evidence of different charm hadronisation mechanisms between $\mathrm{e}^{+} \mathrm{e}^{-}$and pp collisions. The $\Lambda_{\mathrm{c}}^{+} / \mathrm{D}^{0}$ ratio is better described by a model with colour reconnection beyond the leading colour approximation [2], a statistical hadronisation model with an augmented set of charm baryon states predicted by the relativistic quark model (RQM) [5], or a model relying on hadronisation via coalescence and fragmentation [3]. However, all the models underestimate the $\Xi_{\mathrm{c}}^{0,+} / \mathrm{D}^{0}$ and $\mathrm{BR} \times \Omega_{\mathrm{c}}^{0} / \mathrm{D}^{0}$ ratios, except the Catania model [3] including charm quark hadronisation via both coalescence and fragmentation, which would indicate a partonic system similar to a quark-gluon plasma (QGP) in pp collisions.

\section{Charm production and fragmentation in pp collisions}

The charm fragmentation fraction $f\left(\mathrm{c} \rightarrow \mathrm{H}_{\mathrm{c}}\right)$ shown in Fig. 2 (left) represents the probability of a charm quark hadronising into a given charm hadron. The fragmentation fraction for the $\Xi_{\mathrm{c}}^{0}$ baryon is the first measurement in any collision system. An increase of about a factor of 3.3 for the fragmentation fraction for the $\Lambda_{\mathrm{c}}^{+}$baryon with respect to $\mathrm{e}^{+} \mathrm{e}^{-}$and ep collisions, and a corresponding decrease of about a factor of 1.2-1.4 for the $\mathrm{D}^{0}$ meson are observed, showing that the assumption of the charm fragmentation universality (collision-system independence) is broken. Charm quarks hadronise into baryons almost $40 \%$ of the time, which is four times more often than what was measured at colliders with electron beams. 

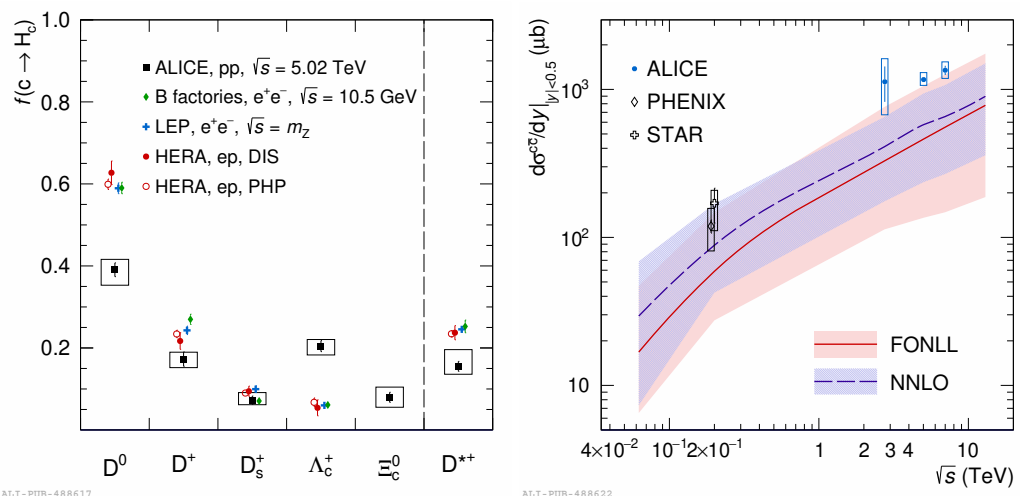

Figure 2. Left: Charm-quark fragmentation fractions into charm hadrons measured in pp collisions at $\sqrt{s}=5.02 \mathrm{TeV}$ in comparison with experimental measurements performed in $\mathrm{e}^{+} \mathrm{e}^{-}$and ep collisions [18]. Right: Charm production cross section at midrapidity per unit of rapidity as a function of the collision energy at the LHC [18] and RHIC [14, 15] compared to FONLL [16] and NNLO [17] calculations.

The cic production cross section per unit of rapidity at midrapidity $\left(\mathrm{d} \sigma^{\mathrm{c} \overline{\mathrm{c}}} /\left.\mathrm{d} y\right|_{|y|<0.5}\right)$ is calculated by summing the $p_{\mathrm{T}}$-integrated cross sections of all measured ground-state charm hadrons $\left(\mathrm{D}^{0}, \mathrm{D}^{+}, \mathrm{D}_{\mathrm{s}}^{+}, \Lambda_{\mathrm{c}}^{+}, \Xi_{\mathrm{c}}^{0}\right.$ and their charge conjugates). The contribution of $\Xi_{\mathrm{c}}^{0}$ is multiplied by a factor of 2 in order to account for the contribution of $\Xi_{c}^{+}$. Since the absence of a $\Omega_{\mathrm{c}}^{0}$ production measurement at hadron colliders, an asymmetric systematic uncertainty is assigned assuming a contribution equal to the one of $\Xi_{\mathrm{c}}^{0}$ considering the prediction of the Catania model [3]. The resulting cc̄ production cross section per unit of rapidity at midrapidity is $\mathrm{d} \sigma^{\mathrm{c} \overline{\mathrm{c}}} /\left.\mathrm{d} y\right|_{|y|<0.5} ^{\mathrm{pp}, 5.02 \mathrm{TeV}}=1165 \pm 44(\mathrm{stat})_{-101}^{+134}(\mathrm{syst}) \mu \mathrm{b}$. The updated fragmentation fractions obtained in pp collisions at $\sqrt{s}=5.02 \mathrm{TeV}$ allow the recomputation of the charm production cross sections per unit of rapidity at midrapidity in pp collisions at $\sqrt{s}=2.76$ and $7 \mathrm{TeV}$, which are about $40 \%$ higher than the previously published results $[12,13]$. The measured $\mathrm{c} \overline{\mathrm{c}}$ production cross section per unit of rapidity at midrapidity together with measurements at RHIC [14, 15] are located at the upper edge of FONLL [16] and NNLO [17] predictions.

\section{Charm hadronisation in $\mathrm{p}-\mathrm{Pb}$ collisions}

Charm hadronisation in $\mathrm{p}-\mathrm{Pb}$ collisions at $\sqrt{s_{\mathrm{NN}}}=5.02 \mathrm{TeV}$ is also investigated. The left panel of Fig. 3 shows the $\Lambda_{\mathrm{c}}^{+} / \mathrm{D}^{0}$ ratio as a function of $p_{\mathrm{T}}$ in pp and $\mathrm{p}-\mathrm{Pb}$ collisions at 5.02 $\mathrm{TeV}$. This ratio is measured down to $p_{\mathrm{T}}=0$ in $\mathrm{p}-\mathrm{Pb}$ collisions for the first time at the LHC. The measurements of $\Lambda_{\mathrm{c}}^{+} / \mathrm{D}^{0}$ in $\mathrm{pp}$ and $\mathrm{p}-\mathrm{Pb}$ collisions are qualitatively consistent with each other, although a larger ratio in $3<p_{\mathrm{T}}<8 \mathrm{GeV} / c$ and a lower ratio in $1<p_{\mathrm{T}}<2 \mathrm{GeV} / c$ are measured in $\mathrm{p}-\mathrm{Pb}$ collisions with respect to $\mathrm{pp}$ collisions. A clear decreasing trend with increasing $p_{\mathrm{T}}$ is obtained in both pp and $\mathrm{p}-\mathrm{Pb}$ collisions for $p_{\mathrm{T}}>2 \mathrm{GeV} / c$. The nuclear modification factor $R_{\mathrm{pPb}}$ of the $\Lambda_{\mathrm{c}}^{+}$baryon measured as a function of $p_{\mathrm{T}}$ together with $R_{\mathrm{pPb}}$ of non-strange $\mathrm{D}$ mesons are shown in the right panel of Fig. 3 . There is a significant suppression for $p_{\mathrm{T}}<2 \mathrm{GeV} / c$ and enhancement for $4<p_{\mathrm{T}}<8 \mathrm{GeV} / c$ in $\mathrm{p}-\mathrm{Pb}$ collisions with respect to pp collisions, which is similar as the $p_{\mathrm{T}}$ distribution of $\Lambda_{\mathrm{c}}^{+} / \mathrm{D}^{0}$ ratio, suggesting the presence of possible radial flow effects or a further modification of the charm hadronisation mechanism in p-Pb collisions. The measurement of $R_{\mathrm{pPb}}$ for the $\Lambda_{\mathrm{c}}^{+}$baryon is compared to model calculations. The POWHEG [19] + PYTHIA6 simulations use the POWHEG event generator 

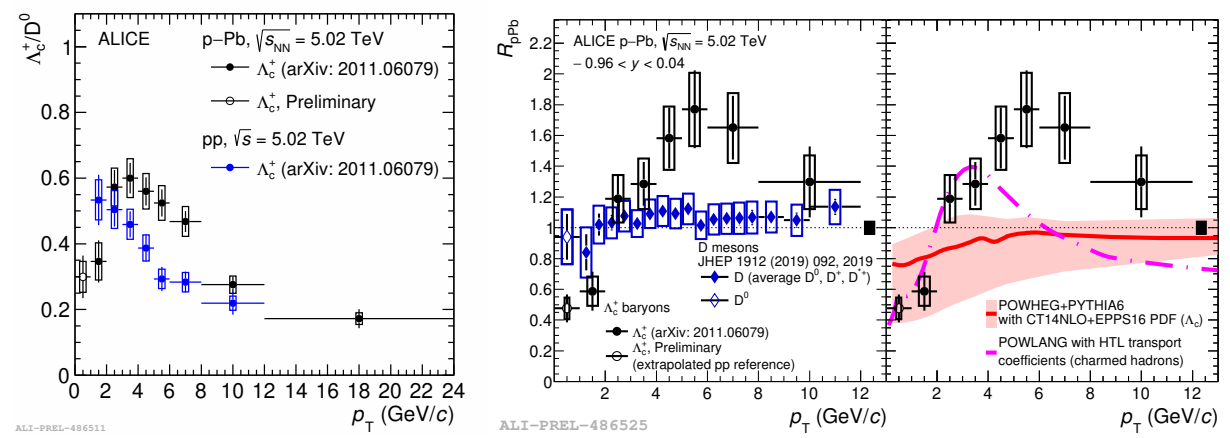

Figure 3. Left: The $\Lambda_{\mathrm{c}}^{+} / \mathrm{D}^{0}$ ratio as a function of $p_{\mathrm{T}}$ measured in pp collisions and $\mathrm{p}-\mathrm{Pb}$ collisions at $5.02 \mathrm{TeV}$ [7]. Right: The nuclear modification factor $R_{\mathrm{pPb}}$ of the $\Lambda_{\mathrm{c}}^{+}$baryon as a function of $p_{\mathrm{T}}$ in $\mathrm{p}-\mathrm{Pb}$ collisions at $\sqrt{s_{\mathrm{NN}}}=5.02 \mathrm{TeV}$, compared to $R_{\mathrm{pPb}}$ of D mesons, as well as to model expectations [7].

with PYTHIA6 parton shower and EPPS16 [20] parameterisation of the nuclear modification of the PDFs. The POWLANG model [21] assumes that a hot deconfined medium is formed in $\mathrm{p}-\mathrm{Pb}$ collisions. The two models capture some features of the data, but neither of them can quantitatively reproduce the data in the measured $p_{\mathrm{T}}$ interval.

\section{Acknowledgments}

This work is supported by the international postdoctoral exchange fellowship program of Helmholtz Association and the Office of China Postdoc Council (No. 20181016).

\section{References}

[1] J.C. Collins, D.E. Soper, G.F. Sterman, Nucl. Phys. B 263, 37 (1986)

[2] J.R. Christiansen, P.Z. Skands, JHEP 08, 003 (2015)

[3] V. Minissale, S. Plumari, V. Greco (2020), arXiv:2012.12001 [hep-ph]

[4] J. Song, H.h. Li, F.l. Shao, Eur. Phys. J. C 78, 344 (2018)

[5] M. He, R. Rapp, Phys. Lett. B 795, 117 (2019)

[6] S. Acharya et al. (ALICE), JHEP 05, 220 (2021)

[7] S. Acharya et al. (ALICE) (2020), arXiv:2011.06079 [nucl-ex]

[8] S. Acharya et al. (ALICE) (2021), arXiv:2105.05187 [nucl-ex]

[9] S. Acharya et al. (ALICE) (2021), arXiv:2105.05616 [nucl-ex]

[10] Y.K. Hsiao et al., Eur. Phys. J. C 80, 1066 (2020)

[11] P. Skands, S. Carrazza, J. Rojo, Eur. Phys. J. C 74, 3024 (2014)

[12] B. Abelev et al. (ALICE), JHEP 07, 191 (2012)

[13] S. Acharya et al. (ALICE), Eur. Phys. J. C 77, 550 (2017)

[14] L. Adamczyk et al. (STAR), Phys. Rev. D 86, 072013 (2012)

[15] A. Adare et al. (PHENIX), Phys. Rev. C 84, 044905 (2011)

[16] M. Cacciari et al., JHEP 10, 137 (2012)

[17] D. d'Enterria, A.M. Snigirev, Phys. Rev. Lett. 118, 122001 (2017)

[18] S. Acharya et al. (ALICE) (2021), arXiv:2105.06335 [nucl-ex]

[19] S. Frixione, P. Nason, G. Ridolfi, JHEP 09, 126 (2007)

[20] K.J. Eskola et al., Eur. Phys. J. C 77, 163 (2017)

[21] A. Beraudo et al., JHEP 03, 123 (2016) 